\title{
Screening of selected Indonesian plants for antiplatelet activity
}

\author{
NANANG FAKHRUDIN ${ }^{1, \bullet}$, FATIYA FARIH MUFINNAH ${ }^{2}$, MUHAMMAD FAISHAL HUSNI ${ }^{2}$, \\ ARIEF EKA WARDANA ${ }^{2}$, ERADHIAN IRMA WULANDARI ${ }^{2}$, ARBIE RISTANTO PUTRA ${ }^{2}$, \\ DJOKO SANTOSA ${ }^{1}$, ARIEF NURROCHMAD ${ }^{3}$, SUBAGUS WAHYUONO ${ }^{1}$ \\ ${ }^{1}$ Department of Pharmaceutical Biology, Faculty of Pharmacy, Universitas Gadjah Mada. Jl. Sekip Utara, Sleman 55281, Yogyakarta, Indonesia. \\ Tel.+62-274 543120, `email: nanangf@ugm.ac.id \\ ${ }^{2}$ Program of Pharmaceutical Sciences, Faculty of Pharmacy, Universitas Gadjah Mada. Jl. Sekip Utara, Sleman 55281, Yogyakarta, Indonesia \\ ${ }^{3}$ Department of Pharmacology and Clinical Pharmacy, Faculty of Pharmacy, Universitas Gadjah Mada. Jl. Sekip Utara, Sleman 55281, Yogyakarta, \\ Indonesia
}

Manuscript received: 21 August 2021. Revision accepted: 9 November 2021.

\begin{abstract}
Fakhrudin N, Mufinnah FF, Husni MF, Wardana AE, Wulandari EI, Putra AR, Santoso D, Nurrichmad A, Wahyunono S. 2021. Screening of selected Indonesian plants for antiplatelet activity. Biodiversitas 22: 5268-5273. Cardiovascular diseases remain the leading cause of death worldwide. Platelet aggregation plays a crucial role in the development of cardiovascular diseases. Thus, the discovery of bioactive compounds targeting platelet aggregation is a promising approach for combating cardiovascular diseases. Indonesia has plants diversity with the potential to be developed as antiplatelet agents. Thus, this study was aimed to identify the antiplatelet potential of selected Indonesian plant extracts. The plant extracts $\left(100 \mu \mathrm{g} \mathrm{mL}^{-1}\right)$ were evaluated for their antiplatelet activity in $\mathrm{CaCl}_{2}$-induced platelet aggregation in 96-well plates. In an aggregometer, the active extracts were further evaluated in adenosine diphosphate (ADP)-induced platelet aggregation. The antiplatelet agents, aspirin, and ticagrelor were used as reference drugs in the respective screening assays. Of the 139 tested extracts, ten demonstrated antiplatelet activity in $\mathrm{CaCl}_{2}$-induced platelet aggregation. The evaluation of the ten active extracts in ADP-induced platelet aggregation revealed four active extracts. The most active extract was the methanol extract of Cinnamomum sintoc bark, followed by the methanol extract of Leea aequata leaf, and the dichloromethane and methanol extracts of Physalis angulata petal with moderate activities. This study provides essential scientific evidence for the development of antiplatelet agents from plant origins.
\end{abstract}

Keywords: Adenosine diphosphate, cardiovascular diseases, herbal medicine, platelet aggregation, thrombosis

\section{INTRODUCTION}

Cardiovascular disease is the leading cause of death worldwide. In 2019, approximately 17.9 million people died from this disease, and the number of suffered patients rises annually (Virani et al. 2021; WHO 2021). The main feature of cardiovascular disease is atherothrombosis, in which platelet aggregation plays a crucial role. Therefore, inhibition of platelet aggregation is the main target in cardiovascular disease pharmacotherapy (Yeung et al. 2018; Nording et al. 2020). Rested platelets with a biconvex discoid shape change into a fully spread star-like form that tends to aggregate due to an increase in intracellular calcium level. Further, the platelets immediately aggregate in response to the binding of fibrinogen with glycoprotein IIb/IIIa receptors (Ghoshal and Bhattacharyya 2014; Chu et al. 2021). The aggregated platelets release platelet activators, such as thromboxane $\mathrm{A}_{2}$, adenosine diphosphate (ADP), and serotonin, which intensify platelet aggregation, leading to clot formation, thrombus, and vascular blockage (Yun et al. 2016; Rubenstein and Yin 2018). Therefore, inhibition of platelet aggregation serves as a promising therapeutic target in treating cardiovascular diseases (Pimentel et al. 2003; Toyoda et al. 2018).

Currently, the most favorable drug inhibitors of platelet aggregation (antiplatelet) are aspirin and thienopyridines (prasugrel, clopidogrel, and ticlopidine). However, the long-term use of those drugs is limited by the risk of bleeding, drug resistance, and response variability (Di Minno et al. 2011; Ray 2014; Hidayati et al. 2017). Therefore, the search for new antiplatelet agents is a promising approach for combating cardiovascular diseases (Nording et al. 2020; Thomas and Nicolson 2021). For decades, plants have inspired the development of many drugs benefiting human health (Atanasov et al. 2021; Yumni et al. 2021). Indonesia has a vast plant diversity, providing abundant natural compounds that can be explored as antiplatelet agents. This study was aimed to test the antiplatelet activity of 139 selected Indonesian plant extracts. The active extracts can be further developed as antiplatelet agents for the treatment of cardiovascular diseases.

\section{MATERIALS AND METHODS}

\section{Procedures}

Plant extracts

The extracts evaluated in this study were obtained from the Department of Pharmaceutical Biology, Faculty of Pharmacy, Universitas Gadjah Mada (UGM), Yogyakarta, Indonesia. The origin plant materials of the extracts were 
authenticated by a botanist (Dr. Djoko Santosa), and the certificate of authentication was documented in the library of the Faculty of Pharmacy, UGM. All extracts were produced by maceration of the plant materials using ethanol, methanol, dichloromethane, chloroform, water, and ethyl acetate, and the extracts were preserved in the deposit fridge $\left(-20^{\circ} \mathrm{C}\right)$ in the Research Laboratory of the Department of Pharmaceutical Biology, Faculty of Pharmacy, UGM.

\section{Chemicals}

Sodium citrate, aspirin, ticagrelor, bovine serum albumin, Hepes, Tyrode's buffer, Giemsa dye, and adenosine diphosphate were purchased from SigmaAldrich (USA). DMSO, dichloromethane, chloroform, and calcium chloride were obtained from Merck (Germany). Methanol, ethanol, and aquadest were procured from Brataco (Indonesia).

\section{Human participants and platelet source}

Human platelet-rich plasma (PRP) and platelet-poor plasma (PPP) were obtained from healthy donors who met the inclusion and exclusion criteria (Cattaneo et al. 2013) as follows: men/women aged 18-40 years having a platelet number of (15-40) $10^{4} \mu \mathrm{L}^{-1}$, not pregnant, no caffeine consumption, and smoking at least 120 and 30 min before blood withdrawal, respectively, no consumption of drugs affecting hemostasis (e.g., NSAIDs, anticoagulant, or antiplatelet agents) at least 7 days before blood withdrawal, and no hemophilia or bleeding disorders. In addition, all participants received informed consent, and the institutional ethics committee approved the method of the Faculty of Medicine, Public Health, and Nursing, UGM, Yogyakarta (number KE/FK/622/EC/2015).

\section{Platelet preparation}

Human platelets from healthy individuals were prepared as previously described (Fakhrudin et al. 2019; Hastuti et al. 2021). Blood from the donors was added with sodium citrate solution (10\%) and centrifuged (1000 rpm, $15 \mathrm{~min}$ ). The upper phase supernatant (PRP) was separated, and the lower phase was further centrifuged (3500 rpm, 15 min). Next, the upper supernatant (PPP) was separated. The number of platelets in the PRP was counted, and only the platelet number of (15-40) $10^{4} \mu \mathrm{L}^{-1}$ was used for the experiment.

\section{$\mathrm{CaCl}_{2}$-induced platelet aggregation assay}

The antiplatelet screening assay was performed as previously described (Pimentel et al. 2003) in a 96-well plate. The extracts were tested at 0.5 and $2 \mathrm{mg} \mathrm{mL}^{-1}$. PRP was resuspended $(1: 5 \mathrm{v} / \mathrm{v})$ in a Hepes-Tyrode buffer containing $1 \%$ bovine serum albumin and then incubated at $37^{\circ} \mathrm{C}$ for $5 \mathrm{~min}$. The extracts $(2.5 \mu \mathrm{L}$ in $\mathrm{DMSO})$ were added to the platelet suspension $(192 \mu \mathrm{L})$ and incubated for 30 min. Aspirin and DMSO were used as positive and negative controls, respectively. Platelet aggregation was induced by adding $250 \mathrm{mM} \mathrm{CaCl} 2(4 \mu \mathrm{L})$ followed by 30 min incubation. Each well was added with Giemsa stain (4 $\mu \mathrm{L}$ ) and incubated for $5 \mathrm{~min}$. The solution was gently discarded, and platelet aggregation was characterized by the formation of violet gel in the well. The extract that was able to inhibit violet gel formation was considered active. This experiment was performed in a triplicate.

\section{ADP-induced platelet aggregation assay}

The second bioassay for confirming the antiplatelet activity of the extracts was conducted using ADP-induced platelet aggregation in an aggregometer instrument (Chrono-Log 490 2D; USA) (Fakhrudin et al. 2020). The extracts were tested at a concentration of $100 \mu \mathrm{g} \mathrm{mL}-1$. DMSO was used as a solvent vehicle or negative control, whereas ticagrelor $\left(10 \mu \mathrm{g} \mathrm{mL}^{-1}\right)$ was used as a reference drug or positive control. PRP $(495 \mu \mathrm{L})$ was added with the extracts $\left(100 \mu \mathrm{g} \mathrm{mL}^{-1}\right.$ in DMSO) and then incubated $\left(37^{\circ} \mathrm{C}\right.$ for $3 \mathrm{~min}$ ) with constant stirring (1200 rpm). In brief, 2.5 $\mu \mathrm{L}$ of $10 \mu \mathrm{M}$ ADP was added to induce platelet aggregation, and the aggregation was recorded for $6 \mathrm{~min}$. The degree of platelet aggregation was normalized to the PPP and calculated based on the amplitude of the curve generated in the aggregometer (Wiyono et al. 2018).

\section{Data analysis}

The data obtained from the ADP-induced platelet aggregation assay were analyzed in GraphPad Prism 8 software, and the statistical analysis was conducted using ANOVA followed by Dunnet post-hoct test.

\section{RESULTS AND DISCUSSION}

This explorative study aimed to find antiplatelet agents from plant origins. Platelet aggregation has a complex pathway with many proteins involved. Thus, two bioassays were employed to screen active plant extracts. The $\mathrm{CaCl}_{2}-$ induced platelet aggregation, recommended by Pimentel et al. (2003) is a fast and general method to evaluate antiplatelet activity. The active extracts obtained from the first method were evaluated in the second method utilizing ADP-induced platelet aggregation (Fakhrudin et al. 2020). ADP induces platelet aggregation by binding to P2Y12 receptor. Using these two bioassays, two pathways of platelet aggregation were targeted.

Results from the first antiplatelet screening assay showed that 10 out of the 139 plant extracts were active by inhibiting the platelet aggregation at 2 and $10 \mathrm{mg} \mathrm{mL}^{-1}$. The ethanol extract of Averrhoa bilimbi leaf (12), methanol extract of Cinnamomun sintoc bark (26), ethanol extract of Garcinia mangostana rind (50), methanol extract of Leea aequata leaf (68), ethanol and dichloromethane extracts of Physalis angulata petal (85 and 86), ethanol extract of Piper cubeba fruit (93), methanol extract of Rubus chrysophyllus leaf and stem (104), and methanol and dichloromethane extracts of Tetracera maingayi leaf and stem (136 and 137) inhibited platelet aggregation in $\mathrm{CaCl}_{2}$ induced platelet aggregation. As expected, aspirin, a clinically used antiplatelet drug, showed potent activity. This result suggested that the method is suitable for the detection of antiplatelet agents. 
Table 1. The results of antiplatelet screening of the plant extracts in $\mathrm{CaCl}_{2}$-induced platelet aggregation assay

\begin{tabular}{|c|c|c|c|c|c|c|}
\hline & & & & & $\begin{array}{r}\text { Plat } \\
\text { aggreg }\end{array}$ & $\begin{array}{l}\text { elet } \\
\text { ation }\end{array}$ \\
\hline Local name & Plant species & Family & Part of plar & t Solvent & $\begin{array}{l}10 \mathrm{mg} \\
\mathrm{mL}^{-1}\end{array}$ & $\begin{array}{l}2 \mathrm{mg} \\
\mathrm{mL}^{-1}\end{array}$ \\
\hline Bawang putih & Allium sativum L. & Amaryllidaceae & $\mathrm{t}$ & DCM & + & + \\
\hline Bawang putih & Allium sativum $\mathrm{L}$. & Amaryllidaceae & $\mathrm{t}$ & $\mathrm{Aq}$ & + & + \\
\hline Bawang putih & Allium sativum $\mathrm{L}$. & Amaryllidaceae & 1 & $\mathrm{EtOH}$ & + & + \\
\hline Bawang putih & Allium sativum L. & Amaryllidaceae & 1 & DCM & + & + \\
\hline Sambiloto & Andrographis paniculata (Burm.f.) Nees & Acanthaceae & $1, \mathrm{~s}$ & $\mathrm{EtOH}$ & + & + \\
\hline Seledri & Apium graveolens $\mathrm{L}$. & Apiaceae & 1 & EtOH & - & + \\
\hline Akar kuning & Arcangelisia flava (L.) Merr. & Menispermaceae & $\mathrm{s}$ & $\mathrm{MeOH}$ & - & + \\
\hline Sukun & Artocarpus altilis (Parkinson ex F.A. Zorn) Fosberg & Moraceae & 1 & $\mathrm{EtOH}$ & + & + \\
\hline Sukun & Artocarpus altilis (Parkinson ex F.A. Zorn) Fosberg & Moraceae & 1 & $\mathrm{Aq}$ & + & + \\
\hline Sukun & Artocarpus altilis (Parkinson ex F.A. Zorn) Fosberg & Moraceae & 1 & EtOAc & + & + \\
\hline Belimbing wuluh & Averrhoa bilimbi L. & Oxalidaceae & 1 & DCM & + & + \\
\hline Belimbing wuluh & Averrhoa bilimbi $\mathbf{L}$. & Oxalidaceae & l & EtOH & - & - \\
\hline Belimbing wuluh & Averrhoa bilimbi $\mathrm{L}$. & Oxalidaceae & $\mathrm{f}$ & $\mathrm{EtOH}$ & - & + \\
\hline Belimbing wuluh & Averrhoa bilimbi $\mathrm{L}$. & Oxalidaceae & $\mathrm{f}$ & DCM & + & + \\
\hline Belimbing manis & Averrhoa carambola L. & Oxalidaceae & 1 & DCM & + & + \\
\hline Belimbing manis & Averrhoa carambola $\mathrm{L}$. & Oxalidaceae & 1 & $\mathrm{EtOH}$ & - & + \\
\hline Belimbing manis & Averrhoa carambola $\mathrm{L}$. & Oxalidaceae & $\mathrm{f}$ & DCM & + & + \\
\hline Belimbing manis & Averrhoa carambola L. & Oxalidaceae & $\mathrm{f}$ & $\mathrm{EtOH}$ & + & + \\
\hline Asam riang & Begonia coriacea Hassk. & Begoniaceae & $\mathrm{s}, \mathrm{l}, \mathrm{fl}$ & $\mathrm{CHCl}_{3}$ & - & + \\
\hline Asam riang & Begonia coriacea Hassk. & Begoniaceae & $\mathrm{s}, \mathrm{l}, \mathrm{fl}$ & $\mathrm{MeOH}$ & + & + \\
\hline Rotan sega & Calamus caesius Bl. & Arecaceae & $\mathrm{h}$ & $\mathrm{CHCl}_{3}$ & + & + \\
\hline Rotan sega & Calamus caesius Bl. & Arecaceae & $\mathrm{h}$ & $\mathrm{MeOH}$ & - & + \\
\hline Daun teh & Camellia sinensis (L.) O.K. & Theaceae & 1 & $\mathrm{EtOH}$ & + & + \\
\hline Tali putri & Cassytha filiformis $\mathrm{L}$. & Lauraceae & $1, \mathrm{~s}$ & $\mathrm{MeOH}$ & + & + \\
\hline Tali putri & Cassytha filiformis $\mathrm{L}$. & Lauraceae & $1, \mathrm{~s}$ & DCM & + & + \\
\hline Sintok & Cinnamomum sintoc $\mathbf{B l}$. & Lauraceae & c & $\mathrm{MeOH}$ & - & - \\
\hline Sintok & Cinnamomum sintoc $\mathrm{Bl}$. & Lauraceae & $\mathrm{c}$ & $\mathrm{CHCl}_{3}$ & - & + \\
\hline Kayu manis Sri Lanka & Cinnamomum zeylanicum Bl. & Lauraceae & $\mathrm{c}$ & EtOH & + & + \\
\hline Galing kerbau & Cissus adnata Roxb. & Vitaceae & $1, \mathrm{~s}, \mathrm{f}, \mathrm{fl}$ & $\mathrm{MeOH}$ & + & + \\
\hline Galing kerbau & Cissus adnata Roxb. & Vitaceae & $\mathrm{l}, \mathrm{s}, \mathrm{f}, \mathrm{fl}$ & DCM & + & + \\
\hline Galing kerbau & Cissus adnata Roxb. & Vitaceae & $1, \mathrm{~s}, \mathrm{f}$ & $\mathrm{MeOH}$ & + & + \\
\hline Irah-irah & Cissus javana DC. & Vitaceae & $1, \mathrm{~s}$ & $\mathrm{MeOH}$ & - & + \\
\hline Senggugu & Clerodendron serratum (L.) Moon & Lamiaceae & $\mathrm{r}$ & $\mathrm{EtOH}$ & + & + \\
\hline Kunyit & Curcuma longa $\mathrm{L}$. & Zingiberaceae & $\mathrm{rh}$ & $\mathrm{EtOH}$ & + & + \\
\hline Kunyit & Curcuma longa $\mathrm{L}$. & Zingiberaceae & rh & $\mathrm{Aq}$ & - & + \\
\hline Temu putih & Curcuma zedoaria Roxb. & Zingiberaceae & $\mathrm{t}$ & $\mathrm{EtOH}$ & + & + \\
\hline Temu putih & Curcuma zedoaria Roxb. & Zingiberaceae & rh & $\mathrm{EtOH}$ & + & + \\
\hline Wortel & Daucus carota L. & Apiaceae & $\mathrm{t}$ & $\mathrm{EtOH}$ & + & + \\
\hline Rigu ripung & Erechtites minima (Poir.) DC. & Compositae & $1, \mathrm{~s}$ & $\mathrm{MeOH}$ & - & + \\
\hline Rigu ripung & Erechtites minima (Poir.) DC. & Compositae & $1, \mathrm{~s}$ & $\mathrm{CHCl}_{3}$ & + & + \\
\hline Daun dadap serep & Erythrina subumbrans (Hassk.) Merr. & Leguminosae & $\mathrm{r}, \mathrm{s}, \mathrm{l}$ & $\mathrm{CHCl}_{3}$ & + & + \\
\hline Daun dadap serep & Erythrina subumbrans (Hassk.) Merr. & Leguminosae & $\mathrm{r}, \mathrm{s}, \mathrm{l}$ & $\mathrm{MeOH}$ & - & + \\
\hline Teklan & Eupatorium riparium $\mathrm{Reg}$. & Compositae & $1, \mathrm{~s}$ & $\mathrm{CHCl}_{3}$ & + & + \\
\hline Teklan & Eupatorium riparium $\mathrm{Reg}$. & Compositae & $1, \mathrm{~s}$ & $\mathrm{MeOH}$ & - & + \\
\hline Jirak & Eurya nitida Korth. & Pentaphylacaceae & $\overrightarrow{1}, \mathrm{~s}$ & $\mathrm{MeOH}$ & - & + \\
\hline Pasak bumi & Eurycoma longifolia Jack. & Simaroubaceae & $\mathrm{r}$ & $\mathrm{MeOH}$ & + & + \\
\hline Awar-awar & Ficus septica Burm.f. & Moraceae & 1 & $\mathrm{EtOH}$ & + & + \\
\hline Akar karamaha & Ficus sagittata Vahl. & Moraceae & $1, \mathrm{~s}$ & $\mathrm{CHCl}_{3}$ & + & + \\
\hline Akar karamaha & Ficus sagittata Vahl. & Moraceae & $1, \mathrm{~s}$ & $\mathrm{MeOH}$ & + & - \\
\hline Manggis & Garcinia mangostana $\mathbf{L}$. & Clusiaceae & ri & EtOH & - & - \\
\hline Kapulaga ambon & Globba marantina L. & Zingiberaceae & $\mathrm{h}$ & $\mathrm{MeOH}$ & + & + \\
\hline Kapulaga ambon & Globba marantina $\mathrm{L}$ & Zingiberaceae & $\mathrm{h}$ & $\mathrm{CHCl}_{3}$ & + & + \\
\hline Daun dewa & Gynura procumbens (Lour.) Merr. & Compositae & 1 & $\mathrm{EtOH}$ & + & + \\
\hline Daun dewa & Gynura procumbens (Lour.) Merr. & Compositae & 1 & DCM & - & + \\
\hline Sirihan & Heckeria peltata (L.) Kunth. & Piperaceae & $1, \mathrm{~s}$ & $\mathrm{CHCl}_{3}$ & + & + \\
\hline Sirihan & Heckeria peltata (L.) Kunth. & Piperaceae & $1, \mathrm{~s}$ & $\mathrm{MeOH}$ & + & + \\
\hline Tumpak babi & Hornstedtia mollis (Bl.) Val. & Zingiberaceae & $\mathrm{rh}$ & $\mathrm{MeOH}$ & + & + \\
\hline Tumpak babi & Hornstedtia mollis (Bl.) Val. & Zingiberaceae & rh & $\mathrm{CHCl}_{3}$ & + & + \\
\hline Buah naga & Hylocereus costaricensis (F.A.C.Weber) Britton \& Rose & Cactaceae & $\mathrm{f}$ & $\mathrm{EtOH}$ & + & + \\
\hline Bunga sapa & Impatiens platypetala Lindl. & Balsaminaceae & $\mathrm{ae}$ & $\mathrm{CHCl}_{3}$ & + & + \\
\hline Bunga sapa & Impatiens platypetala Lindl. & Balsaminaceae & ae & $\mathrm{MeOH}$ & - & + \\
\hline Ubi jalar & Ipomoea batatas (L.) Lam. & Convolvulaceae & $\mathrm{t}$ & $\mathrm{EtOH}$ & + & + \\
\hline Daun gatal & Laportea aestuans (L.) Chew. & Urticaceae & $1, \mathrm{~s}, \mathrm{r}$ & $\mathrm{CHCl}_{3}$ & + & + \\
\hline Daun gatal & Laportea aestuans (L.) Chew. & Urticaceae & $1, \mathrm{~s}, \mathrm{r}$ & $\mathrm{MeOH}$ & + & + \\
\hline Daun gatal & Laportea stimulans (L.f.) Miq. & Urticaceae & $\mathrm{l}, \mathrm{s}, \mathrm{r}$ & $\mathrm{CHCl}_{3}$ & + & + \\
\hline Daun gatal & Laportea stimulans (L.f.) Miq. & Urticaceae & $1, \mathrm{~s}, \mathrm{r}$ & $\mathrm{MeOH}$ & - & + \\
\hline Mali-mali putih & Leea aequata $\mathrm{L}$. & Vitaceae & $1, \mathrm{~s}$ & $\mathrm{CHCl}_{3}$ & + & + \\
\hline Mali-mali putih & Leea aequata $\mathrm{L}$. & Vitaceae & $\mathbf{l}, \mathrm{s}$ & МeOH & - & - \\
\hline
\end{tabular}




\begin{tabular}{|c|c|c|c|c|c|c|}
\hline Ambulia & Limnophila rugosa (Roth.) Merr. & Plantaginaceae & $1, \mathrm{~s}$ & DCM & + & + \\
\hline Ambulia & Limnophila rugosa (Roth.) Merr. & Plantaginaceae & $1, \mathrm{~s}$ & $\mathrm{MeOH}$ & + & + \\
\hline Sanrego & Lunasia amara Blanco & Rutaceae & $1, \mathrm{~s}$ & $\mathrm{MeOH}$ & + & + \\
\hline Apel & Malus domestica Borkh. & Rosaceae & $\mathrm{f}$ & $\mathrm{EtOH}$ & + & + \\
\hline Mangga kasturi & Mangifera casturi Kosterm. & Anacardiaceae & $\mathrm{f}$ & $\mathrm{MeOH}$ & - & + \\
\hline Senggani & Marumia affinis Korth. & Melastomaceae & $1, \mathrm{~s}$ & DCM & + & + \\
\hline Senggani & Marumia affinis Korth. & Melastomaceae & $1, \mathrm{~s}$ & $\mathrm{MeOH}$ & + & + \\
\hline Mengkudu & Morinda citrifolia $\mathrm{L}$ & Rubiaceae & $\mathrm{f}$ & $\mathrm{EtOH}$ & + & + \\
\hline Kemuning & Murraya paniculata (L.) Jack. & Rutaceae & $\mathrm{t}$ & $\mathrm{EtOH}$ & + & + \\
\hline Kemangi & Ocimum basilicum L. forma citratum Back. & Lamiaceae & 1 & $\mathrm{EtOH}$ & + & + \\
\hline Daun kumis kucing & Orthosiphon aristatus (B1.) Miq. & Lamiaceae & 1 & $\mathrm{EtOH}$ & + & + \\
\hline Ginseng & Panax notoginseng (Burkill) F.H.Chen & Araliaceae & $\mathrm{r}$ & $\mathrm{EtOH}$ & + & + \\
\hline Meniran & Phyllanthus niruri L. & Phyllanthaceae & $1, \mathrm{~s}$ & DCM & + & + \\
\hline Meniran & Phyllanthus niruri L. & Phyllanthaceae & $1, \mathrm{~s}$ & $\mathrm{EtOH}$ & + & + \\
\hline Ciplukan & Physalis angulata $\mathrm{L}$. & Solanaceae & $\mathrm{f}$ & $\mathrm{EtOH}$ & - & + \\
\hline Ciplukan & Physalis angulata L. & Solanaceae & f & DCM & - & + \\
\hline Ciplukan & Physalis angulata $\mathbf{L}$. & Solanaceae & $\mathbf{p}$ & EtOH & - & - \\
\hline Ciplukan & Physalis angulata $\mathrm{L}$. & Solanaceae & p & DCM & - & - \\
\hline Poh-pohan & Pilea melastomoides (Poir) Bl. & Urticaceae & $1, \mathrm{~s}, \mathrm{r}, \mathrm{fl}$ & $\mathrm{CHCl}_{3}$ & + & + \\
\hline Poh-pohan & Pilea melastomoides (Poir) Bl. & Urticaceae & $1, \mathrm{~s}, \mathrm{r}, \mathrm{fl}$ & $\mathrm{MeOH}$ & + & + \\
\hline Sirih hitam & Piper acre Blume & Piperaceae & $1, \mathrm{~s}$ & $\mathrm{CHCl}_{3}$ & + & + \\
\hline Sirih hitam & Piper acre Blume & Piperaceae & $1, \mathrm{~s}$ & $\mathrm{MeOH}$ & - & + \\
\hline Sirih hutan & Piper caducibracteum C. DC. & Piperaceae & 1 & $\mathrm{CHCl}_{3}$ & + & + \\
\hline Kemukus & Piper cubeba L.f. & Piperaceae & $\mathrm{f}$ & DCM & + & + \\
\hline Kemukus & Piper cubeba L.f. & Piperaceae & $\mathbf{f}$ & EtOH & - & - \\
\hline Cabe jawa & Piper retrofractum Vahl. & Piperaceae & f & EtOH & + & + \\
\hline Jering & Archidendron ellipticum (Blanco) I.C.Nielsen & Leguminosae & $1, \mathrm{~s}$ & $\mathrm{CHCl}_{3}$ & - & + \\
\hline Jering & Archidendron ellipticum (Blanco) I.C.Nielsen & Leguminosae & $1, \mathrm{~s}$ & $\mathrm{MeOH}$ & - & + \\
\hline Daun sendok & Plantago lanceolata L. & Plantaginaceae & 1 & DCM & + & + \\
\hline Daun sendok & Plantago lanceolata L. & Plantaginaceae & 1 & $\mathrm{EtOH}$ & + & + \\
\hline Daun sendok & Plantago major L. & Plantaginaceae & 1 & $\mathrm{EtOH}$ & + & + \\
\hline Matoa & Pometia pinnata J.R.Forst. \& G.Forst. & Sapindaceae & 1 & DCM & + & + \\
\hline Matoa & Pometia pinnata J.R.Forst. \& G.Forst. & Sapindaceae & 1 & $\mathrm{EtOH}$ & + & + \\
\hline Buas-buas, Singkil & Premna cordifolia Roxb. & Lamiaceae & 1 & $\mathrm{EtOH}$ & + & + \\
\hline Jambu biji & Psidium guajava $\mathrm{L}$ & Myrtaceae & $\mathrm{f}$ & $\mathrm{EtOH}$ & + & + \\
\hline Beberetean, Arben & Rubus chrysophyllus Reinw. Ex Miq. & Rosaceae & $1, \mathrm{~s}$ & МeОН & - & - \\
\hline Beberetean, Arben & Rubus chrysophyllus Reinw. Ex Miq. & Rosaceae & $1, \mathrm{~s}$ & $\mathrm{CHCl}_{3}$ & + & + \\
\hline Akar kayu raung & Schefflera scandens (Bl.) R. Vig. & Araliaceae & $1, \mathrm{~s}$ & $\mathrm{CHCl}_{3}$ & - & + \\
\hline Akar kayu raung & Schefflera scandens (Bl.) R. Vig. & Araliaceae & $1, \mathrm{~s}$ & $\mathrm{MeOH}$ & - & + \\
\hline Jakatuwa & Scoparia dulcis L. & Scrophulariaceae & $1, \mathrm{~s}$ & $\mathrm{MeOH}$ & - & + \\
\hline Jakatuwa & Scoparia dulcis L. & Scrophulariaceae & $1, \mathrm{~s}$ & DCM & + & + \\
\hline Daun cakar ayam & Selaginella plana Hieron & Selaginellaceae & $1, \mathrm{~s}, \mathrm{r}$ & $\mathrm{CHCl}_{3}$ & + & + \\
\hline Daun cakar ayam & Selaginella plana Hieron & Selaginellaceae & $1, \mathrm{~s}, \mathrm{r}$ & $\mathrm{MeOH}$ & - & + \\
\hline Sidagori, Saliguri & Sida mysorensis Wight \& Arn. & Malvaceae & $1, \mathrm{~s}, \mathrm{fl}$ & $\mathrm{MeOH}$ & - & + \\
\hline Tomat & Solanum lycopersicum L. & Solanaceae & f & $\mathrm{EtOH}$ & + & + \\
\hline Tempuyung & Sonchus arvensis L. & Compositae & 1 & $\mathrm{EtOH}$ & + & + \\
\hline Kemangi cina & Spigelia anthelmia L. & Loganiaceae & $1, \mathrm{~s}$ & DCM & + & + \\
\hline Kemangi cina & Spigelia anthelmia L. & Loganiaceae & $1, \mathrm{~s}$ & $\mathrm{MeOH}$ & + & + \\
\hline Kedondong & Spondias dulcis Parkinson & Anacardiaceae & 1 & DCM & + & + \\
\hline Kedondong & Spondias dulcis Parkinson & Anacardiaceae & 1 & $\mathrm{EtOH}$ & - & + \\
\hline Kepel & Stelechocarpus burahol (Blume) Hook.f. \& Thomson & Annonaceae & 1 & DCM & - & + \\
\hline Kepel & Stelechocarpus burahol (Blume) Hook.f. \& Thomson & Annonaceae & 1 & $\mathrm{EtOH}$ & + & + \\
\hline Daun hantap & Sterculia oblongata $\mathrm{R} . \mathrm{Br}$. & Sterculiaceae & 1 & $\mathrm{MeOH}$ & + & + \\
\hline Keji beling & Sericocalyx crispus (L.) Bremek. & Acanthaceae & 1 & $\mathrm{EtOH}$ & + & + \\
\hline Mahoni & Swietenia macrophylla King & Meliaceae & se & $\mathrm{EtOH}$ & + & + \\
\hline Jambu air & Syzigium aqueum (Burm.f.) Alston & Myrtaceae & $\mathrm{f}$ & DCM & + & + \\
\hline Jambu air & Syzigium aqueum (Burm.f.) Alston & Myrtaceae & 1 & DCM & + & + \\
\hline Jambu air & Syzigium aqueum (Burm.f.) Alston & Myrtaceae & f & $\mathrm{EtOH}$ & - & + \\
\hline Jambu air & Syzigium aqueum (Burm.f.) Alston & Myrtaceae & 1 & $\mathrm{EtOH}$ & + & + \\
\hline Jamblang & Syzigium cumini (L.) Skeels & Myrtaceae & se & $\mathrm{EtOH}$ & + & + \\
\hline Jamblang & Syzigium cumini (L.) Skeels & Myrtaceae & se & DCM & + & + \\
\hline Jamblang & Syzigium cumini (L.) Skeels & Myrtaceae & 1 & $\mathrm{EtOH}$ & + & + \\
\hline Jamblang & Syzigium cumini (L.) Skeels & Myrtaceae & 1 & DCM & + & + \\
\hline Jamblang & Syzigium cumini (L.) Skeels & Myrtaceae & $\mathrm{f}$ & $\mathrm{EtOH}$ & - & + \\
\hline Jamblang & Syzigium cumini (L.) Skeels & Myrtaceae & $\mathrm{f}$ & DCM & + & + \\
\hline Som Jawa & Talinum paniculatum (Jacq.) Gaertn. & Portulacaceae & $\mathrm{r}$ & DCM & + & + \\
\hline Som Jawa & Talinum paniculatum (Jacq.) Gaertn. & Portulacaceae & $\mathrm{r}$ & $\mathrm{EtOH}$ & + & + \\
\hline Mempelas & Tetracera maingayi Hoogland & Dilleniaceae & $\mathbf{l}, \mathrm{s}$ & $\mathrm{CHCl}_{3}$ & - & - \\
\hline Mempelas & Tetracera maingayi Hoogland & Dilleniaceae & l, $\mathrm{s}$ & МeOH & - & - \\
\hline Bratawali & Tinospora crispa (L.) Hook. f. \& Thomson & Menispermaceae & $\mathrm{f}$ & $\mathrm{EtOH}$ & + & + \\
\hline Bangle & Zingiber montanum (J.Koenig) Link ex A.Dietr. & Zingiberaceae & $\mathrm{rh}$ & $\mathrm{EtOH}$ & + & + \\
\hline Aspirin & & & & & - & - \\
\hline
\end{tabular}


Note: Part of plant. b: bark; f: fruit; fl: flower; h: herb; l: leaf; t: tuber; p: petal; r: root; rh: rhizome; ri: rind; s: stem; se: seed. Solvent. $\mathrm{CHCl}_{3}$ : chloroform; $\mathrm{MeOH}$ : methanol, EtOH: etanol; DCM: dichloromethane; Aq: aquadest; EtOAc: ethyl acetate. Results, + : violet gel presents (aggregation); - : no violet gel (no aggregation). The bold letters indicated the active plant extracts.

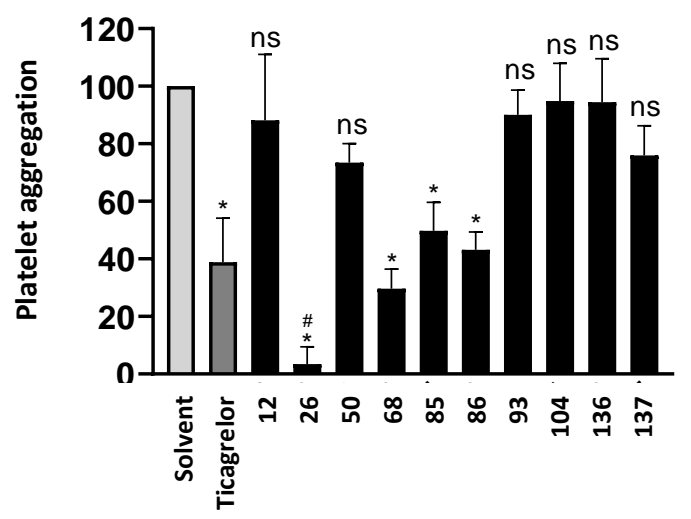

Figure 1. Antiplatelet activity of the selected active extracts on ADP-induced platelet aggregation. Ethanol extract of Averrhoa bilimbi leaf (12), methanol extract of Cinnamomum sintoc bark (26), ethanol extract of Garcinia mangostana rind (50), methanol extract of Leea aequata leaf $(\mathbf{6 8})$, ethanol and dichloromethane extracts of Physalis angulata petal (85 and 86), ethanol extract of Piper cubeba fruit (93), methanol extract of Rubus chrysophyllus leaf and stem (104), and methanol and dichloromethane extracts of Tetracera maingayi leaf and stem (136 and 137) were tested at $100 \mu \mathrm{g} \mathrm{mL}^{-1}$. The solvent (DMSO) and ticagrelor were at $0.1 \%$ and $10 \mu \mathrm{g} \mathrm{mL}^{-1}$, respectively. Data are expressed as mean $\pm \mathrm{SD}$ from three independent experiments; * statistically significant compared with the solvent group, \# statistically significant compared with $68,85,86$ ( $p<0.01$; ANOVA followed by Dunnett's post-hoc test).

Platelet aggregation is also triggered by the activation of platelet receptors, such as P2Y12, PAR2, PAR4, TP $\alpha$, $\alpha 2$, and glocoprotein receptors by their respective ligands. Among these receptors, P2Y12 is the most studied and represents a promising target for antiplatelet agents. Activation of P2Y12 by its ligand ADP not only induces platelet aggregation but also provokes platelet degranulation, leading to additional ADP release that intensifies aggregation (Murugappa and Kunapuli 2006; Yeung et al. 2018). The use of prasugrel and ticagrelor as potent P2Y12 antagonists is effective for atherothrombosis (Collet et al. 2021). Thus, we evaluated the effectivity of the active plant extracts to inhibit platelet aggregation induced by ADP (Figure 1). Ticagrelor inhibited platelet aggregation induced by ADP, indicating that this bioassay was appropriate for the screening of P2Y12 antagonists. The methanol extract of $C$. sintoc cortex (27), methanol extract of $L$. aequata leaf (68), ethanol and dichloromethane extracts of $P$. angulata petal ( 85 and $\mathbf{8 6}$ ) demonstrated antiplatelet activity.

Figure 1 shows that the methanol extract of $C$. sintoc cortex showed the strongest effect among the extracts. The bark of $C$. sintoc contains tannins, 3-allyl-6methoxyphenol, methyl eugenol, cis-methyl isoeugenol, $p$ acetamidophenol, methyl myristate, methyl palmitate, methyl linoleate, methyl octadec-9-enoate, methyl stearate, and methyl 11-eicosenoate (Jantan et al. 2005; Fakhrudin et al. 2019). Interestingly, none of these compounds were reported to have antiplatelet activity. Although a previous study demonstrated that the tannin content of the methanol extract of $C$. sintoc cortex is linearly correlated with the antiplatelet activity induced by epinephrine (Fakhrudin et al. 2019), the compound responsible for the antiplatelet activity remains unknown. Thus, further research on isolating and identifying the antiplatelet compound from $C$. sintoc bark is a vast prospect. Cinnamomum tenuifolium (Dong et al. 2013) and C. philippinense (Yu et al. 1994) contain the antiplatelet agents isotenuifolide and cinnamophilin, respectively. However, no study reported the presence of these compounds in $C$. sintoc.

The other plant extracts with a moderate activity are the methanol extract of $L$. aequata leaf $(\mathbf{6 8})$ and the ethanol and dichloromethane extracts of $P$. angulata petal $(\mathbf{8 5}$ and 86). Leea aequata is a wild shrub traditionally used because of its antimalarial, antifever, antiantelmentic, antijaundice, and astringent effects (Sinaga et al. 2018). A recent study has reported the presence of new compounds, including (7S,8R)9'-O-acetylcedrusin (a lignan) and (3S,4S)-4-chloro-3-hydroxypiperidin-2-one (a lactam), and several known compounds (Tun et al. 2019). However, none of them have antiplatelet activity. Apart from $L$. aequata, $P$. angulata contains physalin $\mathrm{B}$ as an antiplatelet compound isolated from the aerial parts (Mangwala et al. 2013; Hsu et al. 2014; Yang et al. 2018). In line with the previous finding, we found that the petal extracts of $P$. angulata showed antiplatelet activity. Therefore, it merits further study because it is underutilized apart from the edible fruit.

In summary, we identified ten plant extracts showing antiplatelet activity by using $\mathrm{CaCl}_{2}$-induced platelet aggregation. Four out of ten active extracts demonstrated significant antiplatelet activity in ADP-induced platelet aggregation. The methanol extract of $C$. sintoc bark is the most potent extract followed by the methanol extract of $L$. aequata leaf, the dichloromethane and methanol extracts of $P$. angulata petal. These extracts are promising to be developed further as antiplatelet agents to combat cardiovascular diseases. This study provides a scientific basis for the development of antiplatelet agents from natural plant products.

\section{ACKNOWLEDGEMENTS}

The authors thank Miranda Pratiwi and Setiono for their excellent technical assistance. This study was supported by Penelitian Unggulan Perguruan Tinggi, Ministry of Education and Culture, Republic of Indonesia (Grand number: 240/UN1.P.III/DIT-LIT/LT/2017). The data were used by Fatiya Farih Mufinnah, Muhammad Faishal Husni, Arief Eka Wardana, Eradhian Irma Wulandari, and Arbie 
Ristanto Putra for their undergraduate thesis in the Faculty of Pharmacy, Universitas Gadjah Mada. The authors declared no conflict of interest in this study.

\section{REFERENCES}

Atanasov AG, Zotchev SB, Dirsch VM, Orhan IE, Banach M, Rollinge JM, Barreca D, Weckwerth W, Bauer R, Bayer EA et al. 2021. Natural products in drug discovery: Advances and opportunities. Nat Rev Drug Discov 20 (3): 200-216. DOI: 10.1038/s41573-020-00114-

Cattaneo M, Cerletti C, Harrison P, Hayward CP, Kenny D, Nugent D, Nurden P, Rao AK, Schmaier AH, Watson SP, Lussana F, Pugliano MT, Michelson AD. 2013. Recommendations for the standardization of light transmission aggregometry: A consensus of the working party from the platelet physiology subcommittee of SSC/ISTH. J Thromb Haemost 11: 1183-1189. DOI: 10.1111/jth.12231.

Chu Y, Guo H, Zhang Y, Qiao R. 2021. Procoagulant platelets: Generation, characteristics, and therapeutic target. J Clin Lab Anal 35 (5): 1-10. DOI: 10.1002/jcla.23750.

Collet J-P, Thiele H, Barbato E, Barthélémy O, Bauersachs J, Bhatt DL, Dendale P, Dorobantu M, Edvardsen T, Folliguet T et al. 2021. 2020 ESC guidelines for the management of acute coronary syndromes in patients presenting without persistent ST-segment elevation. Eur Heart J 42 (14): 1289-1367. DOI: 10.1093/eurheartj/ehaa575.

Di Minno MND, Guida A, Camera M, Colli S, Di Minno G, Tremoli E. 2011. Overcoming limitations of current antiplatelet drugs: A concerted effort for more profitable strategies of intervention. Ann Med 43 (7): 531-544. DOI: 10.3109/07853890.2011.582137.

Dong HP, Wu HM, Chen SJ, Chen CY. 2013. The effect of butanolides from Cinnamomum tenuifolium on platelet aggregation. Molecules 18 (10): 11836-41. DOI: 10.3390/molecules181011836.

Fakhrudin N, Pertiwi KK, Takubessi MI, Susiani EF, Nurrochmad A, Widyarini S, Sudarmanto A, Nugroho AA, Wahyuono S. 2020. A geranylated chalcone with antiplatelet activity from the leaves of breadfruit (Artocarpus altilis). Pharmacia 67 (4): 173-180. DOI: 10.3897/pharmacia.67.e56788.

Fakhrudin N, Wiyono T, Putra AR, Nurrochmad A, Widyarini S. 2019. The evaluation on anti-platelet and antithrombosis activities of Cinnamomum sintoc bark extract. Thai J Pharm Sci 43 (4): 219-226. DOI: $10.1063 / 1.5062741$

Ghoshal K, Bhattacharyya M. 2014. Overview of platelet physiology: Its hemostatic and nonhemostatic role in disease pathogenesis. Sci World J 2014: 781857. DOI: 10.1155/2014/781857.

Hastuti I, Nurrochmad A, Puspitasari I, Fakhrudin N. 2021. Studi aktivitas antiplatelet dan antitrombosis ekstrak air daun sukun (Artocarpus altilis (Park.) Fosberg). J Tumbuhan Obat Indonesia 14 (1): 85-94. DOI: 10.22435/jtoi.v14i1.4227. [Indonesian]

Hidayati F, Irawan B, Mumpuni H. 2017. Aspirin and clopidogrel resistance in coronary artery disease. Acta Cardio Indones 3 (1): 33-44. DOI: 10.22146/aci.29683.

Hsu CC, Liu PY, Farh L, Tseng W, Yang KC, Wu CC, Chang F. 2014. Investigation and characterization of the antiplatelet activities of physalin B. Intl J Latest Res Sci Tech 3 (6): 114-120.

Jantan Ib, Yalvema MF, Ayop N, Ahmad AS. 2005. Constituents of the essential oils of Cinnamomum sintoc Blume from a mountain forest of Peninsular Malaysia. Flavour Fragr J 20 (6): 601-604. DOI: 10.1002/ffj. 1495

Mangwala PK, Lusakibanza M, Mesia K, Tona L, Tits M, Angenot L, Frédérich M, Van Meervelt L. 2013. Isolation, pharmacological activity and structure determination of physalin B and $5 \beta, 6 \beta$ epoxyphysalin B isolated from Congolese Physalis angulata L. Acta $\begin{array}{lllll}\text { Crystallogr } & \mathrm{C} & 69 & (12): & 1557-1562 .\end{array}$ 10.1107/S010827011303117X.

Murugappa S, Kunapuli SP. 2006. The role of ADP receptors in platelet function. Front Biosci 11: 1977-1986. DOI: 10.2741/1939.

Nording H, Baron L, Langer HF. 2020. Platelets as therapeutic targets to prevent atherosclerosis. Atherosclerosis 307: 97-108. DOI: 10.1016/j.atherosclerosis.2020.05.018.

Pimentel SMV, Bojo ZP, Roberto AVD, Lazaro JEH, Mangalindan GC, Florentino LM, Lim-Navarro P, Tasdemir D, Ireland CM, Concepcion GP. 2003. Platelet aggregation inhibitors from Philippine marine invertebrate samples screened in a new microplate assay. Mar Biotechnol 5 (4): 395-400. DOI: 10.1007/s10126-002-0080-3.

Ray S. 2014. Clopidogrel resistance: The way forward. Indian Heart J 66 (5): 530-534. DOI: 10.1016/j.ihj.2014.08.012.

Rubenstein DA, Yin W. 2018. Platelet-activation mechanisms and vascular remodeling. In: Pollock DM (eds.). Comprehensive Physiology. Am J Physiol 8 (3): 1117-1156 DOI: 10.1002/cphy.c170049.

Sinaga E, Ginting N, $\quad$ Suwarso E. $2018 . \quad$ Uji aktivitas antikejang ekstrak etanol daun Titanus (Leea Aequata L.) terhadap ileum Marmut terpisah (Cavia porcellus) secara in vitro. Talenta Conf Ser 1 (1): 320-330. DOI: 10.32734/tm.v1i1.66. [Indonesian]

Thomas MR, Nicolson PLR. 2021. Inhibiting novel mechanisms of thrombosis: Next-generation antiplatelet therapy. Platelets 32 (1):5-6. DOI: 10.1080/09537104.2020.1853422.

Toyoda T, Isobe K, Tsujino T, Koyata Y, Ohyagi F, Watanabe T, Nakamura M, Kitamura Y, Okudera H, Nakata K et al. 2018. Direct activation of platelets by addition of $\mathrm{CaCl}(2)$ leads coagulation of platelet-rich plasma. Intl J Implant Dent 4 (1): 23-23. DOI: 10.1186/s40729-018-0134-6.

Tun NL, Hu DB, Xia MY, Zhang DD, Yang J, Oo TN, Wang YH, Yang XF. 2019. Chemical constituents from ethanoic extracts of the aerial parts of Leea aequata $\mathrm{L}$., a traditional folk medicine of myanmar. Nat Prod Bioprospect 9 (3): 243-249. DOI: 10.1007/s13659-019-0209-y.

Virani SS, Alonso A, Aparicio HJ, Benjamin EJ, Bittencourt MS, Callaway CW, Carson AP, Chamberlain AM, Cheng S, Delling FN et al. 2021. Heart disease and stroke Statistics-2021 update: A report from the American Heart Association. Circulation 143 (8): e254-e743. DOI: 10.1161/CIR.0000000000000950.

WHO. 2021. Cardiovascular diseases (CVDs) [Internet]. Geneva, Switzerland WHO. Available at https://www.who.int/en/newsroom/fact-sheets/detail/cardiovascular-diseases-(cvds)

Wiyono T, Nurrochmad A, Widyarini S, Fakhrudin N. 2018. The tannin content and anti platelet-aggregation activity of Cinnamomum sintoc extract. AIP Conf Proc 2021 (1): 1-6. DOI: 10.1063/1.5062741.

Yang Y, Yi L, Wang Q, Xie B, Sha C, Dong Y. 2018. Physalin B suppresses inflammatory response to lipopolysaccharide in RAW264.7 cells by inhibiting NF-кB signaling. J Chem 2018: 1-6. DOI: $10.1155 / 2018 / 7943140$.

Yeung J, Li W, Holinstat M. 2018. Platelet signaling and disease: Targeted therapy for thrombosis and other related diseases. Pharmacol Rev 70 (3): 526-548. DOI: 10.1124/pr.117.014530.

Yu SM, Ko FN, Wu TS, Lee JY, Teng CM. 1994. Cinnamophilin, a novel thromboxane $\mathrm{A}_{2}$ receptor antagonist, isolated from Cinnamomum philippinense. Eur J Pharmacol 256 (1): 85-91. DOI: 10.1016/00142999(94)90620-3.

Yumni GG, Widyarini S, Fakhrudin N. 2021. Kajian etnobotani, fitokimia, farmakologi dan toksikologi sukun (Artocarpus altilis (Park.) Fosberg). J Tumbuhan Obat Indonesia 14 (1): 48-63. DOI: 10.22435/jtoi.v14i1.3944. [Indonesian]

Yun SH, Sim EH, Goh RY, Park JI, Han JY. 2016. Platelet activation: The mechanisms and potential biomarkers. BioMed Res Int 2016: 15. DOI: 10.1155/2016/9060143. 